\title{
TOWARD A BROADER SYSTEMS APPROACH TO RESOURCE MANAGEMENT - AN UTOPIAN VISION TO GUIDE REALISTS
}

\author{
Jagdeep Singh \\ Patrik Baard \\ KTH Royal Institute of Technology, Sweden
}

\begin{abstract}
This paper proposes an ideal/utopian normative vision on sustainable resource management with its twofold aim to highlight; the need to explicate and address the underlying evaluative and normative assumptions in industrial ecology (IE) research (theoretical challenges), and the challenges to implement such broader system level solutions (practical challenges). The main theoretical challenges highlighted in the paper are the lack of linkages between natural science, social science and normative analysis. The main practical challenges remain the issues regarding mutual trust and, governance and regulation in a global context. The normative vision aims to propose actions to develop (current) realistic approaches to resource management.
\end{abstract}

\section{KEYWORDS}

Sustainable resource management, global challenges, social embeddedness, industrial ecology, utopian/idea vision, normative approach

\section{INTRODUCTION TO THE PAPER}

For the last two decades (or so), industrial ecology has emerged as a new field to develop perspectives and solutions to ecological consequences of industrial activities. However, IE being a social construct [1], suffers heavy criticisms and disputes over the issues of conceptual duality leading to compromised solutions [2]. Industrial ecology solutions have contributed to address different sustainability issues at various scales ranging from local contamination to resource management globally. Yet, to target the root causes of sustainability challenges such as (over)consumption, IE research have to tread a long path ahead by framing broad questions recognizing social dimension and their implications to solutions (Figure 1). Recently, Boons and Howard-Grenvile [3] have highlighted the questions major questions regarding social context in which material and energy exchanges are enabled/constrained/governed in IE solutions - cognition, culture, structural and political embeddedness in IE.

Solving these issues at once by any discipline is hard; nonetheless, at first hand, an increased diffusion of ideologies among different disciplines can highlight the important issues which 
should be recognized while proposing long-term solutions to sustainability challenges. As a starting, the core symbolic and physical system - interface of economy and techno-sphere (Figure 1) - must be understood in a broad sense with whole society and environment [2]. Further, to develop a missing normative rationale as a framework to discuss these challenges in a global context. To address this gap, we propose a broader systems approach to resource management - an ideal/utopian vision to guide resource management efforts at various levels.

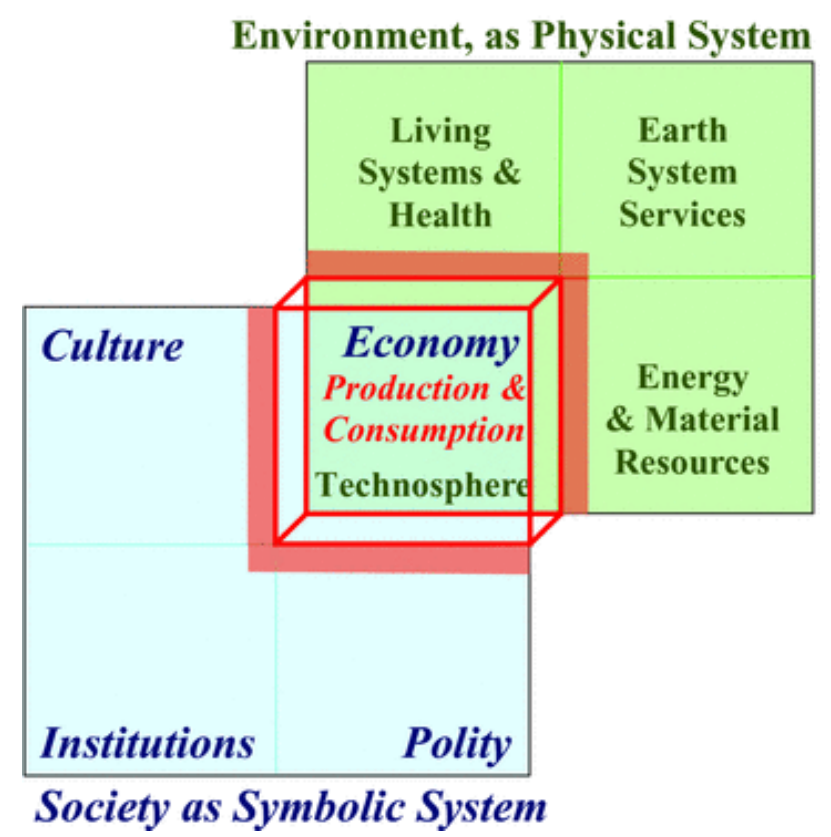

Figure 1. Social and natural science to be combined into IE. Source: [2]

The proposed ideal/utopian rationale is based on systems thinking and sustainability science [4] strategies summarized by Ness and colleagues [5] as follows:

- "covering the range of spatial scales between diverse phenomena,

- accounting for temporal inertias and urgency of processes,

- $\quad$ dealing with functional complexity resulting from multiple stresses, and

- $\quad$ the recognition of a wide range of outlooks equating to usable knowledge in both science and society."

The vision highlights a need for incorporating various scales and levels of analysis [6] to satisfy normative considerations regarding why goals should be set?, how to distribute responsibility? and who should guide such efforts?. Need to recognize the multitudes of perspectives, cross-scale dynamics and actors' interactions have been highlighted in a broader system - global spatial scale of production and consumption with long-term temporal scales to address issues such as equity and environmental justice. Fundamental challenges such as a lack of; mutual goal, trust and, governance and regulation, to realize such a vision have been discussed. Further, these issues put in broader context to exemplify problems of current value system (growth imperative and assumptions regarding human behavior), normative reasons for regulation of resource use on a global level and burden sharing (externalities and compliance). Following issues have been highlighted and discussed under this vision: 
- Not enough or little attention to address the main causes of the problem often rooted outside the WM system.

- System level expansion: economy-wide product design approaches which integrate broad environmental objectives in the product design processes.

o Resource discard mechanisms - consumer level

o Issues of scales and levels

- Link between WM and, production and consumption: At the WM system end, most of the efforts have significantly been focused on removing the waste as being generated and treating it with the available best technologies within various economic and environmental constrains. However, too little consideration is offered to the broader goals such as what institutional changes are needed outside the boundary of WM system to sustainably implement the WM hierarchy.

- Understanding of consumption dynamics in current economic structures: consumption based economic growth driven by hegemony of desires through advertisements.

- Lack of a shared vision on what is sustainable resource management?; how it should be approached?; and what it should include?: silos of science, differing actors' perceptions on roles and responsibilities, consumers' awareness, mutual trust.

\section{REFERENCES}

[1]. Cohen-Rosenthal, E., A Walk on the Human Side of Industrial Ecology. American Behavioral Scientist, 2000. 44(2): p. 245-264.

[2]. Huppes, G., M. Ishikawa, and G.J. Kramer, Challenges for Industrial Ecology in Practice and Theory. Journal of Industrial Ecology, 2011. 15(5): p. 677-679.

[3]. Boons, F. and J. Howard-Grenvile, Introducing the social embeddedness of industrial ecology in The Social Embeddedness of Industrial Ecology. 2009, Edward Elgar: UK. p. 304.

[4]. Kates, W.R., et al., Sustainability Science, in Science. 2001. p. 641-642.

[5]. Ness, B., S. Anderberg, and L. Olsson, Structuring problems in sustainability science: The multi-level DPSIR framework. Geoforum, 2010. 41(3): p. 479-488.

[6]. Cash, D.W., et al., Scale and cross-scale dynamics: governance and information in a multilevel world. Ecology and Society, 2006. 11(2). 